\title{
Caracterización de la entomofauna acuática en cuatro quebradas de la cuenca del río San Juan, Chocó, Colombia
}

\author{
Zuleyma Mosquera-Murillo*, Karen E. Córdoba-Aragón \\ Grupo de Limnología, Facultad de Ciencias Básicas, Universidad Tecnología del Chocó, Chocó, Colombia
}

\begin{abstract}
Resumen
Se estudió la entomofauna acuática de cuatro quebradas de la cuenca del río San Juan en el departamento del Chocó (Colombia). Entre febrero y julio de 2007 se recolectaron 2.377 especímenes distribuidos en nueve órdenes, 35 familias y 52 géneros. El orden Ephemeroptera fue el más representativo en términos de abundancia $(61,09 \%)$ y de riqueza (11 géneros), seguido de Coleoptera (13,38 \%), con 12 géneros. El índice de diversidad de ShannonWeaver, de 2,95 bits/ind, así como la riqueza total de géneros (52) encontrada en las quebradas, demuestran su gran diversidad, con diferencias significativas entre ellas, pero no en cuanto a los meses de muestreo. De acuerdo con el índice de Jaccard, basado en la presencia de diferentes géneros identificados, se observó que las quebradas Profundo y Colorado presentaron mucha similitud $(0,65)$, mientras que el mayor valor de disimilitud lo presentó la quebrada San Francisco $(0,48)$. Los parámetros físicos y químicos estaban dentro de los rangos normales y presentaron poca variación, lo que indica que las quebradas tienen buenas condiciones ecológicas para el establecimiento de una comunidad acuática diversa.
\end{abstract}

Palabras clave: entomofauna acuática, San Juan, Chocó.

Characterization of aquatic entomologic fauna in four streams of San Juan river (Chocó - Colombia)

\begin{abstract}
The aquatic entomofauna of four streams of the San Juan river in the department of Chocó (Colombia) was studied. Between February and July 2007, 2,377 specimens belonging to nine orders, 35 families and 52 genera were collected. The order Ephemeroptera was the most representative in terms of abundance $(61.09 \%)$ and biological richness (11 genera), followed by the order Coleoptera (13.38\%). A Shannon-Weaver diversity index of 2.95 and the total number of genera found in the streams showed their high diversity; we found significant differences between them, but not between sampling months. According to the Jaccard index based on the presence of different identified genera, we observed that the Profundo and Colorado streams had high similarity (0.65), while the highest value of dissimilarity was found in the San Francisco stream (0.48). The physical and chemical parameters were within normal ranges and showed little variation, indicating that the streams have good ecological conditions for the establishment of a diverse aquatic community.
\end{abstract}

Key words: Aquatic entomofauna, San Juan, Chocó, Colombia.

\section{Introducción}

El objetivo principal del estudio ecológico de los sistemas lóticos es entender los mecanismos y procesos responsables de las diferencias y similitudes entre las comunidades y su relación con las características fisicoquímicas del agua donde se desarrollan (Machado \& Roldán, 1981). Entre las comunidades que habitan estos sistemas se encuentran los macroinvertebrados acuáticos, que dan cuenta de gran parte de su diversidad biológica y con frecuencia son el principal componente animal de los sistemas lóticos (Esteves, 1988).

Los macroinvertebrados acuáticos, y en general los insectos, juegan un papel importante en todos los procesos ecológicos de los sistemas acuáticos, son un enlace importante para la transferencia de energía a diversos niveles tróficos de las cadenas alimentarias acuáticas (Malmqvist, et al., 2004), aceleran los procesos de descomposición de detritos y contribuyen al reciclaje de nutrientes (Wotton \& Malmqvist, 2001; Hanson, et al., 2010); además, consumen gran cantidad de algas y otros microorganismos asociados con el perifiton en los ríos o con el plancton en los lagos y, muchas veces, este consumo aumenta la productividad primaria, ya que se elimina el tejido poco productivo y se mineralizan los nutrientes (Wallace \& Webster, 1996; Allan \& Castillo, 2007).

\footnotetext{
*Correspondencia:

Zuleyma Mosquera-Murillo, zuleymamosquera@gmail.com

Recibido: 20 de noviembre de 2014

Aceptado: 2 de marzo de 2015
} 
Los cambios ambientales tienen gran influencia en la distribución, abundancia y riqueza de las comunidades de insectos acuáticos en los cuerpos de agua (Hawkins, et al., 1982, Ometo, et al., 2000, Shieh et al., 2000, Ocón, et al., 2004), las cuales actúan como testigos biológicos del nivel de deterioro ambiental de las corrientes superficiales, ya que reflejan las condiciones y los cambios ecológicos que ocurren en el sistema (Alba-Tercedor, 1996).

Entre los cambios que se pueden detectar con los insectos acuáticos están las alteraciones del hábitat y los cambios de temperatura, sustrato y concentración de desechos domésticos e industriales en función del tiempo de ocurrencia e intensidad del evento (Rosenberg, et al., 1993a). Estas características posibilitan su uso como herramientas para la evaluación espacio-temporal de los cambios en la calidad del agua de cuerpos de agua superficiales mediante el seguimiento biológico (Rosenberg, et al., 1993b).

Los siguientes son algunos de los trabajos que brindan aportes valiosos al conocimiento de los insectos acuáticos en Colombia: Zamora (2002), Posada \& Roldán (2003), Muñoz (2004), Guevara, et al., (2004, 2005, 2007), Molano, et al., (2005), Romero, et al., (2006), Latorre, et al., (2006), Rojas, et al., (2006), Reinoso, et al., (2007, 2008), Vásquez \& Reinoso (2012), Vásquez, et al., (2014), Forero, et al., (2013, 2014), Zúñiga, et al., (2014), Parra, et al., (2014). Específicamente en el Chocó, los estudios publicados se han desarrollado en la cuenca del río Atrato (Rivas, et al., 2003; Torres, et al., 2006; Asprilla, et al., 2006; Mosquera, et al., 2006; Casas, et al., 2006, Córdoba, et al., 2007, Salas, et al., 2011), pero no hay información sobre la cuenca del río San Juan. En el presente estudio se caracterizó la comunidad de insectos acuáticos de cuatro quebradas pertenecientes a la cuenca del río San Juan, todas con características de primer orden, como una contribución al conocimiento de la diversidad de los macroinvertebrados acuáticos de la región.

\section{Materiales y métodos}

\section{Area de estudio}

El estudio se llevó cabo en cuatro quebradas pertenecientes a la cuenca del río San Juan. Este río, ubicado entre los $5^{\circ}$ $16^{\prime} 10^{\prime \prime}$ de latitud norte y los $76^{\circ} 73^{\prime} 10^{\prime \prime}$ de longitud oeste, es uno de los más importantes del Chocó; cubre un área total de $15.000 \mathrm{~km}^{2}$ y tiene una longitud de $750 \mathrm{~km}$ (Bedoya, et al., 2009). Se encuentra a una altura de $75 \mathrm{msnm}$, con temperaturas de entre 27 y $29{ }^{\circ} \mathrm{C}$ y una precipitación anual promedio que alcanza valores medios de alrededor de 10.000 $\mathrm{mm} / \mathrm{año} \mathrm{(Instituto} \mathrm{de} \mathrm{Investigaciones} \mathrm{Ambientales} \mathrm{del}$ Pacífico - IIAP, 2013).

En el área del San Juan predominan las zonas de vida de bosque pluvial tropical (bp-T) y bosque muy húmedo tropical (bmh-T) (Holdridge, 1996). Las quebradas estudiadas presentaban aguas transparentes y un sustrato compuesto de piedras, arena y hojarascas en descomposición, con caudal de entre 0,067 y $3,76 \mathrm{~m}^{3} \mathrm{seg}^{-1}$, y una amplia cobertura vegetal en sus márgenes como resultado de la escasa influencia antrópica en la zona.

\section{Muestreo y análisis}

Se hicieron cinco salidas de campo entre febrero y julio de 2007, durante las cuales se seleccionó un tramo representativo de 100 metros en cada una de las quebradas para evaluar las condiciones fisicoquímicas y recolectar muestras de insectos acuáticos. En cada quebrada se midieron in situ el oxígeno disuelto $\left(\mathrm{mg} / \mathrm{l}^{-1}\right)$, el $\mathrm{pH}$ (unidades de $\left.\mathrm{pH}\right)$, la temperatura del agua $\left({ }^{\circ} \mathrm{C}\right)$, la conductividad eléctrica $(\mu \mathrm{S} /$ $\mathrm{cm})$ y los sólidos totales disueltos $\left(\mathrm{mg} / \mathrm{l}^{-1}\right)$ con ayuda de un equipo digital mutiparámetros $(\mathrm{HACH})$; la alcalinidad total $\left(\mathrm{mg} / \mathrm{l}^{-1} \mathrm{CaCO}_{3}\right)$ se midió en el Laboratorio de Limnología de la Universidad Tecnológica del Chocó siguiendo las recomendaciones de los Standard Methods for the Examination of Water and Wastewater (American Public Health Association - APHA, 1992).

La recolección de los insectos acuáticos se hizo con diferentes métodos de captura de acuerdo al sustrato presente: una red D-net para la vegetación marginal y pinzas entomológicas para los organismos presentes en piedras, troncos y hojarasca. Se hizo el mismo esfuerzo de muestreo en cada uno de los sustratos presentes (30 minutos). Los organismos se fijaron en alcohol al $70 \%$ en frascos previamente rotulados y luego se trasladaron al Laboratorio de Limnología de la Universidad Tecnológica del Chocó para su determinación taxonómica con ayuda de un estereoscopio y claves especializadas para cada uno de los taxones (Merrit \& Cummins, 1996; Fernández \& Domínguez, 2001; Posada \& Roldan, 2003; Domínguez \& Fernández, 2009; Springer, et al., 2010).

Para describir la comunidad de macroinvertebrados en las diferentes quebradas, se emplearon los parámetros biológicos de diversidad biológica de Shannon - Wiener $\left(\mathrm{H}^{\prime}\right)$, la dominancia de Simpson (D) y la riqueza total (programa Past, versión 1.57, Hammer, et al., 2001); sus diferencias espaciales (quebradas) y temporales (muestreos) se evaluaron mediante análisis de varianza (ANOVA), previa revisión de las condiciones de normalidad y homocedasticidad. Se empleó el índice de Jaccard para comparar el grado de similitud entre las quebradas. Para estos análisis se empleó el programa Minitab, versión 15.

\section{Resultados y discusión}

\section{Aspectos fisicos y quimicos}

La tabla 1 resume los valores de las variables físicas y químicas de las cuatro quebradas estudiadas. El oxígeno disuelto se mantuvo por encima de los $5 \mathrm{mg} / \mathrm{l}^{-1}$ y no superó los $9 \mathrm{mg} / \mathrm{l}^{-1}$, valores que favorecen una alta densidad biológica y el desarrollo de la entomofauna acuática (Guerrero \& Manjares, 2003). El pH se mantuvo dentro del rango de la neutralidad, lo cual es propio de ecosistemas oligotróficos (Roldan, 1992), e indican una estabilidad alta del medio 
Tabla 1. Valores promedio de algunas variables físicas y químicas medidas en cuatro cuerpos de agua de la cuenca del San Juan, Chocó Colombia

\begin{tabular}{|c|c|c|c|c|c|c|}
\hline \multirow[t]{2}{*}{ Variables / Quebradas } & Profundo P1 & Profundo P2 & Colorado & Buena Vida & San Francisco & \multirow[t]{2}{*}{$\mathrm{CV}$} \\
\hline & \multicolumn{5}{|c|}{ Promedios } & \\
\hline Oxígeno disuelto (mg/l) & 6,21 & 5,4 & 5,01 & 5,48 & 5,98 & 18,17 \\
\hline pH (unidades de pH) & 7,2 & 7,7 & 7,01 & 7,88 & 7,02 & 7,28 \\
\hline Temperatura del agua $\left({ }^{\circ} \mathrm{C}\right)$ & 26,18 & 26,51 & 26,52 & 25,53 & 25,85 & 2,44 \\
\hline Conductividad eléctrica $(\mu \mathrm{S} / \mathrm{cm})$ & 29,75 & 33,25 & 20,07 & 33,4 & 40,7 & 28,11 \\
\hline Sólidos totales disueltos (mg/l) & 14,83 & 16,62 & 10 & 16,68 & 20,35 & 28,2 \\
\hline Alcalinidad (mg/l $\left.\mathrm{CaCO}^{3}\right)$ & 7,67 & 15,7 & 10,4 & 12,18 & 18,18 & 37,56 \\
\hline
\end{tabular}

acuático. La temperatura del agua no superó los $27^{\circ} \mathrm{C}$ en ninguno de los cuerpos de agua estudiados, manteniéndose dentro del rango registrado para la zona tropical, con valores entre 25 y $30{ }^{\circ} \mathrm{C}$ (Asprilla, et al., 1998). La conductividad eléctrica, al igual que la alcalinidad y los sólidos totales disueltos, presentaron promedios dentro de los rangos citados por Roldán (1992) para ecosistemas acuáticos de tierras bajas, es decir, entre 30 y $60 \mu \mathrm{S} / \mathrm{cm}$ para el caso de la conductividad y menores de $100 \mathrm{mg} / \mathrm{l}^{-1}$ para la alcalinidad. Se presentaron pocas variaciones entre los cuerpos de agua en cuanto a las variables medidas, a excepción de la alcalinidad, con un coeficiente de variación de $37,56 \%$.

\section{Entomofauna acuática}

Se recolectaron 2.377 especímenes (correspondientes todos a estados inmaduros de los insectos), los cuales se agruparon en nueve órdenes, 35 familias y 52 géneros (Tabla 2). Estos datos son muy significativos para el país, puesto que son los primeros reportes de insectos acuáticos de la cuenca del río San Juan en el Chocó. El orden Ephemeroptera presentó la mayor densidad de individuos, con 1.452 (61,09\%), siendo uno de los de mayor riqueza biológica, con 11 géneros (Figura 1), hallazgo que coincide con otras investigaciones llevadas a cabo en el país (Arango, et al., 2008; Vásquez-Ramos \& Reinoso, 2012; Forero-Céspedes, et al., 2014), en las que se resalta la abundancia de este orden de insectos acuáticos.

Las ninfas del orden Ephemeroptera constituyen una parte importante de las cadenas alimenticias en ríos y arroyos como alimento para otros organismos acuáticos, procesadores de materia orgánica y herbívoros, además, son elementos importantes en la transferencia de energía dentro del sistema acuático y están presentes en casi todos los tipos de cuerpos de agua, aunque con mayor abundancia y diversidad en ríos y arroyos de fondos rocosos (Flowers \& De la Rosa, 2010). El sustrato rocoso y pedregoso es dominante en las quebradas del río San Juan, lo cual contribuye a la abundancia de este orden.

El mayor número de individuos y de familias (29) se registró en la quebrada Profundo, P1 (655), siendo la Leptophlebiidae la más representativa, seguida por Baetidae. Estas dos familias son las de mayor distribución y diversidad en Suramérica y registran el mayor número de géneros y especies en Colombia (Zúñiga, et al., 2004). A nivel de géneros, Thraulodes fue el más representativo en los cuerpos de agua estudiados, lo que puede atribuirse a las condiciones ecológicas reinantes en ellos, caracterizadas por altos valores de oxígeno disuelto, $\mathrm{pH}$ neutro, baja conductividad y pocos sólidos, pues las ninfas de este género son muy sensibles a su variación. El género Thraulodes es el más común dentro de la familia Leptophlebiidae, habita en todo tipo de ríos, en arroyos, en agua con baja carga orgánica y en un amplio rango de altitudes (Zúñiga, et al., 2004; Domínguez, et al., 2006), y prefiere el sustrato pedregoso (Zúñiga, et al., 2004). Se destaca la presencia del género Hydrosmilodon en la cuenca del río San Juan, el cual fue reportado por primera vez para Colombia en la cuenca del Atrato por Casas, et al., (2006).

El orden Coleoptera (13,38 \%) fue el segundo más importante en el estudio, con ocho familias, entre las que Elmidae y Psephenidae fueron las más abundantes, y se encontraron asociadas a una variedad de sustratos naturales sumergidos en las quebradas estudiadas (piedras, hojarasca, raíces de plantas acuáticas, vegetación ribereña, fondos arenosos, etc.). La mayoría de las familias de este orden presentan especies que viven en ambientes lóticos y que forman parte de las comunidades bentónicas, donde no alcanzan grandes densidades (Fernández \& Domínguez, 2001); sin embargo, presentan una gran diversidad, gracias a que ocupan un amplio espectro de hábitats acuáticos y semiacuáticos, incluidas áreas ripícolas, ecosistemas de agua fría, de corriente rápida, salobres y áreas estancadas de estuarios y ciénagas (Richoux, 1994; Merrit \& Cummins, 1996), como lo corroboran los 12 géneros que se reportan para este orden en la presente investigación.

La familia Elmidae ha sido reportada como abundante en otras investigaciones a nivel nacional (Mosquera, et al., 2002; Caupaz-Flórez, et al., 2006; Arias, et al., 2007; Zúñiga, et al., 2013). Los miembros de esta familia viven en aguas corrientes con alto contenido de oxígeno, destacándose como los más comunes y numerosos de los 
Tabla 2. Abundancia de la entomofauna acuática en cuatro quebradas de la cuenca del río San Juan, Chocó - Colombia

Taxón/Quebrada

\begin{tabular}{|c|c|c|c|c|c|c|c|c|}
\hline Ephemeroptera & & & & & & & & \\
\hline \multirow{4}{*}{ Leptophlebiidae } & Farrodes & 23 & 2 & 144 & 0 & 4 & 173 & 7,28 \\
\hline & Thraulodes & 147 & 174 & 136 & 83 & 194 & 734 & 30,88 \\
\hline & Hydrosmilodon & 21 & 0 & 0 & 0 & 3 & 24 & 1,01 \\
\hline & Terpides & 22 & 13 & 1 & 15 & 2 & 53 & 2,23 \\
\hline \multirow{4}{*}{ Baetidae } & Cloeodes & 11 & 13 & 4 & 1 & 9 & 38 & 1,60 \\
\hline & Americabaetis & 38 & 31 & 24 & 14 & 44 & 151 & 6,35 \\
\hline & Camelobaetidius & 33 & 15 & 1 & 1 & 37 & 87 & 3,66 \\
\hline & Baetodes & 38 & 9 & 9 & 0 & 2 & 58 & 2,44 \\
\hline \multirow{3}{*}{ Leptohyphidae } & Haplohyphes & 2 & 2 & 0 & 0 & 10 & 14 & 0,59 \\
\hline & Leptohyphes & 44 & 29 & 4 & 4 & 23 & 104 & 4,38 \\
\hline & Tricorythodes & 10 & 4 & 0 & 1 & 1 & 16 & 0,67 \\
\hline \multicolumn{9}{|l|}{ Plecoptera } \\
\hline Perlidae & Anacroneuria & 27 & 36 & 32 & 5 & 63 & 163 & 6,86 \\
\hline \multicolumn{9}{|l|}{ Trichoptera } \\
\hline Leptoceridae & Nectosyche & 3 & 2 & 1 & 0 & 9 & 15 & 0,63 \\
\hline Hydropsychidae & Smicridea & 34 & 8 & 32 & 18 & 23 & 115 & 4,84 \\
\hline Phylopotamidae & Chimarra & 2 & 1 & 0 & 7 & 28 & 38 & 1,60 \\
\hline Glossosomatidae & Culoptila & 2 & 2 & 3 & 9 & 2 & 18 & 0,76 \\
\hline Hydrobiosidae & Atopsyche & 3 & 1 & 0 & 0 & 1 & 5 & 0,21 \\
\hline Helicopsychidae & Helicopsyche & 2 & 0 & 1 & 0 & 0 & 3 & 0,13 \\
\hline Calamoceratidae & Phylloicus & 1 & 1 & 0 & 1 & 0 & 3 & 0,13 \\
\hline Odontoceridae & Marilia & 1 & 2 & 0 & 0 & 0 & 3 & 0,13 \\
\hline Hydroptilidae & Ochrotrichia & 1 & 0 & 0 & 0 & 0 & 1 & 0,04 \\
\hline \multicolumn{9}{|l|}{ Odonata } \\
\hline Calopterigydae & Hetaerina & 5 & 1 & 8 & 1 & 1 & 16 & 0,67 \\
\hline \multirow{3}{*}{ Libellulidae } & Macrothemis & 3 & 1 & 0 & 0 & 0 & 4 & 0,17 \\
\hline & Brechmorhoga & 4 & 1 & 1 & 0 & 0 & 6 & 0,25 \\
\hline & Dythemis & 4 & 2 & 0 & 0 & 0 & 6 & 0,25 \\
\hline Cordulidae & Neocordulia & 0 & 0 & 0 & 0 & 1 & 1 & 0,04 \\
\hline Polythoridae & Polythore & 0 & 0 & 0 & 0 & 1 & 1 & 0,04 \\
\hline Platystictidae & Palaemnema & 7 & 3 & 5 & 0 & 8 & 23 & 0,97 \\
\hline Megapodagrionidae & Heteragrion & 6 & 3 & 6 & 0 & 0 & 15 & 0,63 \\
\hline Gomphidae & Agriogomphus & 0 & 0 & 0 & 2 & 0 & 2 & 0,08 \\
\hline Perilestidae & Perissolestes & 0 & 0 & 0 & 3 & 0 & 3 & 0,13 \\
\hline Coenagrionidae & Argia & 2 & 1 & 0 & 2 & 2 & 7 & 0,29 \\
\hline \multicolumn{9}{|l|}{ Coleoptera } \\
\hline Gyrinidae & Andogirus & 1 & 0 & 0 & 0 & 0 & 1 & 0,04 \\
\hline Ptilodactilidae & Anchytarsus & 2 & 1 & 7 & 3 & 15 & 28 & 1,18 \\
\hline
\end{tabular}




\begin{tabular}{|c|c|c|c|c|c|c|c|c|}
\hline \multirow{4}{*}{ Elmidae } & Heterelmis & 36 & 3 & 6 & 0 & 20 & 65 & 2,73 \\
\hline & Cylloepus & 16 & 10 & 19 & 1 & 0 & 46 & 1,94 \\
\hline & Phanocerus & 0 & 0 & 4 & 0 & 1 & 5 & 0,21 \\
\hline & Macrelmis & 14 & 2 & 10 & 0 & 5 & 31 & 1,30 \\
\hline \multirow{2}{*}{ Psephenidae } & Disersus & 0 & 0 & 0 & 0 & 1 & 1 & 0,04 \\
\hline & Psephenus & 31 & 18 & 37 & 4 & 47 & 137 & 5,76 \\
\hline Gyrinidae & Dineurus & 0 & 0 & 1 & 0 & 0 & 1 & 0,04 \\
\hline Curculionidae & Listronotus & 0 & 0 & 1 & 0 & 0 & 1 & 0,04 \\
\hline Staphylinidae & Stenus & 0 & 0 & 1 & 0 & 0 & 1 & 0,04 \\
\hline Hidraenidae & Hidraena & 1 & 0 & 0 & 0 & 0 & 1 & 0,04 \\
\hline \multicolumn{9}{|l|}{ Hemiptera } \\
\hline \multirow{2}{*}{ Naucoridae } & Criphocricos & 5 & 0 & 12 & 0 & 3 & 20 & 0,84 \\
\hline & Ambrysus & 1 & 1 & 0 & 0 & 0 & 2 & 0,08 \\
\hline \multicolumn{9}{|l|}{ Diptera } \\
\hline Simulidae & Simullium & 7 & 8 & 1 & 0 & 15 & 31 & 1,30 \\
\hline Tipulidae & Hexatoma & 1 & 0 & 1 & 2 & 1 & 5 & 0,21 \\
\hline \multirow{2}{*}{ Chironomidae } & Ablabezmyia & 9 & 1 & 1 & 2 & 4 & 17 & 0,72 \\
\hline & Chironomus & 5 & 0 & 0 & 0 & 0 & 5 & 0,21 \\
\hline \multicolumn{9}{|l|}{ Lepidoptera } \\
\hline Crambidae & Petrophila & 9 & 13 & 0 & 2 & 11 & 35 & 1,47 \\
\hline \multicolumn{9}{|l|}{ Neuroptera } \\
\hline Coridalidae & Corydalus & 21 & 9 & 3 & 0 & 11 & 44 & 1,85 \\
\hline No. de individuos & & 655 & 423 & 516 & 181 & 602 & 2377 & 100,00 \\
\hline
\end{tabular}

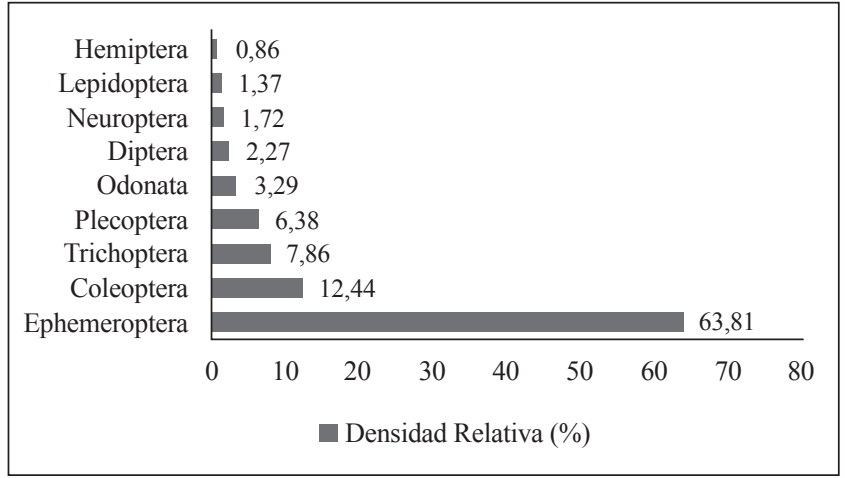

Figura 1. Abundancia relativa de macroinvertebrados acuáticos encontrados en los sistemas estudiados

Byrrhoidea acuáticos (Archangelsky, et al., 2009); los Psephenidae, por su parte, son importantes como indicadores de la calidad del agua y la estabilidad ambiental (Fernández \& Domínguez, 2001).

El orden Trichoptera representó el 8,46\% de los insectos recolectados (201 individuos), con nueve géneros y nueve familias de las 13 reportadas para Colombia por Muñoz
(2004), equivalentes al 20 y 69,23\%, respectivamente, lo que constituye un aporte muy significativo al conocimiento de la diversidad regional y nacional de este orden. Hydropsychidae fue la familia más representativa con 115 individuos (57,21\%), lo que coincide con otras investigaciones realizadas en el departamento del Chocó (Bejarano, et al., 2006; Mosquera \& Bejarano, 2006), y en el país (Guevara, et al., 2005; Rivera-Usme, et al., 2008; Vásquez-Ramos, et al., 2010; Zúñiga, et al., 2013). Los tricópteros viven en aguas corrientes, limpias y oxigenadas, debajo de piedras, troncos y material vegetal; algunas especies habitan en aguas quietas y remansos de ríos y quebradas y son buenas indicadoras de aguas oligotróficas (Domínguez \& Fernández, 2009).

El orden Plecoptera $(6,86 \%)$ se encontró en todos los ecosistemas estudiados, con un total de 163 individuos del género Anacroneuria (Tabla 2). De acuerdo con Zúñiga (2010), este es el género que exhibe la mayor diversidad, abundancia y distribución en los diferentes departamentos y regiones naturales del país, y en el Chocó, su presencia ha sido reportada en diversos cuerpos de agua de la cuenca del río Atrato por Torres, et al., (2006), Asprilla, et al., (2006), Mosquera, et al., (2006) y Salas, et al., (2011), donde sobresale por su abundancia y sensibilidad a la 
contaminación. Estos insectos se encuentran generalmente en aguas con corriente rápida, altos niveles de oxígeno, altos porcentajes de sustratos gruesos en el lecho y presencia de hojarasca (Tamaris-Turizo, et al., 2007; Zúñiga, 2010), y se consideran excelentes bioindicadores de la calidad del agua (Fernández \& Domínguez, 2001).

El orden Odonata $(3,53 \%)$ estuvo representado por 11 géneros y por nueve de las 14 familias reportadas para el país por Álvarez-Arango, et al., (2006), lo que evidencia la gran diversidad de este grupo en la zona, a pesar de su baja densidad. Al respecto, Zúñiga, et al., (2013), señalan en su estudio de quebradas andinas que la vegetación marginal, los sustratos finos y las corrientes lentas son condiciones que favorecen el desarrollo de este grupo de organismos. Las ninfas habitan en diversos hábitats acuáticos y, como depredadores que son, juegan un papel importante en la dinámica de población de sus presas (Lombardo, 1997; Hammond, et al., 2007), constituyen, así, un eslabón importante en la cadena trófica de agua dulce. La familia más abundante del orden es Platystictidae, con el género Palaemnema, que habita quebradas y ríos que corren por bosques, e incluso por áreas con poca vegetación riparia, donde las ninfas se pueden encontrar bajo rocas en el fondo y en lugares de corriente rápida (Ramírez, 2010).

El orden Diptera presentó poca abundancia en las quebradas estudiadas $(2,44 \%)$, representado solo por tres familias, entre las que sobresalió Simulidae como la más abundante (Tabla 2). La poca representatividad que registra este orden contrasta con las investigaciones hechas en el suroccidente colombiano (Chará, et al., 2007, 2009, 2011; Giraldo, et al., 2011) y en quebradas andinas del país (Zuñiga, et al., 2013), en las que es dominante. Esto puede explicarse por el grado de conservación de las quebradas estudiadas, teniendo en cuenta que la abundancia de dípteros se asocia con aguas contaminadas (Abril, et al., 2004). Los simúlidos, por su parte, son propios de aguas oligotróficas, limpias y bien oxigenadas (Rivera, et al., 2008; Domínguez \& Fernández, 2009), y su distribución está influenciada por factores hidráulicos, por el tamaño y la forma del sustrato y la disponibilidad de alimento (Adler, et al., 2004).

Los órdenes Neuroptera $(1,85 \%)$ y Lepidoptera $(1,47 \%)$ fueron los menos abundantes en este estudio y estuvieron representados por las familias Corydalidae y Crambidae, respectivamente. Ambas familias se caracterizan por habitar aguas torrentosas, bien oxigenadas y poco profundas (Roldán, 1996; Roldán, 2003; Domínguez \& Fernández, 2009). Su poca abundancia puede ser el resultado de la poca distribución que presentaron en la zona de estudio, pues solo se encontraron en tres de las quebradas estudiadas. En general, Lepidoptera y Neuroptera tienden a ser grupos minoritarios entre los insectos acuáticos según las investigaciones realizadas en otras zonas del país (Arango, et al., 2008; RiveraUsme, et al., 2008; Walteros-Rodríguez \& Paiba-Alzate, 2010; Zuñiga, et al., 2013).
El orden Hemiptera $(0,93 \%)$ registró una familia, la Naucoridae, con los géneros Criphocricos y Ambrysus. Los miembros de esta familia habitan principalmente en arroyos y ríos pedregosos, entre las rocas del fondo, y son predadores voraces de otros insectos acuáticos (Mazzucconi, et al., 2009). La poca representatividad de este orden contrasta con otras investigaciones como la de Padilla-Gil (2013), en la que se encontró una amplia distribución de este grupo de insectos en el país. En general, los hemípteros muestran preferencias particulares en relación con el microhábitat, como lo son la presencia mayoritaria de macrófitas en el lecho del cauce y el predominio de corrientes lentas, que se relacionan más con ambientes lénticos que con cuerpos de agua corriente (Álvarez \& Roldán, 1983; Mazzucconi, et al., 2009), lo que explica su poca abundancia en la zona, al tratarse de quebradas pequeñas, con poco caudal y muy torrentosas, que son características de la cuenca del río San Juan.

\section{Índices ecológicos}

El índice de diversidad de Shannon-Weiner en la cuenca del río San Juan fue alto $\left(H^{\prime}=2,95\right)$ durante todo el estudio, ya que se considera que valores de $\mathrm{H}^{\prime}$ de 2,70 o más corresponden a un alto grado de diversidad (Margalef, 1998). El valor promedio para este índice fue de 2,03 bits/individuo, siendo la quebrada Profundo, P1, la de mayor diversidad (Tabla 3); hubo diferencias significativas entre las quebradas $(\mathrm{p}=0,011)$, pero no entre los meses de muestreo $(\mathrm{p}=0,548)$. La riqueza total fluctuó entre 3 y 30 taxones, con diferencias significativas entre las quebradas $(\mathrm{p}=0,007)$, pero no entre los meses de muestreo $(\mathrm{p}=0,550)$. La mayor diversidad y riqueza total observadas en la quebrada Profundo, P1 (Tabla 3), puede atribuirse a la mayor cantidad de hábitats que presentaba dicha quebrada y a la presencia de una mayor cobertura vegetal, factores importantes para la distribución de los macroinvertebrados acuáticos. Al respecto, Cardenas, et al., (2007) sostienen que una buena protección de ribera mejora el hábitat, ya que contribuye a tener una mejor calidad de agua, una mayor integridad del cauce y un mejor hábitat físico, lo que incrementa la diversidad y la riqueza de insectos acuáticos bentónicos en las quebradas (Guerrero-Bolaño, et al., 2003; Chara, et al., 2007).

Tabla 3. Índices ecológicos de las cuatro quebradas estudiadas

\begin{tabular}{lccc}
\hline Quebradas/índices & $\begin{array}{c}\text { Diversidad de } \\
\text { Shannon-Wiener }\end{array}$ & $\begin{array}{c}\text { Dominancia } \\
\text { de Simpson }\end{array}$ & $\begin{array}{c}\text { Riqueza } \\
\text { Total }\end{array}$ \\
\hline Profundo P1 & 3,06 & 0,92 & 44 \\
\hline Profundo P2 & 2,39 & 0,80 & 35 \\
Buena Vida & 2,31 & 0,83 & 31 \\
San Francisco & 2,09 & 0,75 & 22 \\
Colorado & 2,61 & 0,86 & 35 \\
\hline
\end{tabular}


El índice de dominancia de Simpson presentó valores relativamente altos en todas las quebradas, con un promedio de 0,77 bits/individuo. La quebrada Profundo, P1, sobresalió con el mayor valor para este índice (Tabla 3). No se observaron diferencias significativas entre los meses de muestreo $(p=0,359)$ ni entre las quebradas $(p=0,092)$. Los valores altos observados para este índice podrían atribuirse a la gran densidad de algunos géneros como Thraulodes, Farrodes (Ephemeroptera) y Anacroneuria (Plecoptera), correspondiente al $45 \%$ de los individuos recolectados, y a su presencia en todas las quebradas estudiadas. Estos géneros presentan un amplio rango de distribución en el Neotrópico (Domínguez, 1999; Domínguez, et al., 2006; Zuñiga, 2010; Zuñiga, et al., 2014) y exhiben una alta sensibilidad a la contaminación y a la degradación del ecosistema acuático (Ribera, et al., 2002), por lo que su abundancia demuestra que los sistemas estudiados presentan un alto grado de conservación.

Las quebradas Profundo y Colorado registraron mayor similitud según el índice de Jaccard $(0,65)$. En las dos había familias que son muy generalistas y que presentan amplios rangos de distribución (Tabla 2); además, ambas presentaban un cauce amplio, vegetación marginal, velocidad de la corriente moderada, una combinación de sustrato pedregoso y arenoso, y gran cantidad de hojarasca, lo cual favorece la presencia de una gran diversidad de insectos acuáticos. De igual manera, sus condiciones fisicoquímicas presentaron similitudes (Tabla 1). La quebrada San Francisco presentó el mayor valor de disimilitud $(0,48)$, posiblemente porque esta se encontraba bastante apartada de las restantes y sus características físicas mostraban marcadas diferencias, como un cauce más angosto y poco profundo, así como una velocidad de corriente más baja.

Con base en los resultados de este estudio preliminar, se puede concluir que las quebradas estudiadas en la cuenca del río San Juan (Chocó) presentan una comunidad de insectos acuáticos muy diversa y compleja, lo que se asocia con sistemas que no han sido alterados, o muy poco, por actividades antropogénicas, y cuya estructura parece estar determinada por la naturaleza del sustrato, la velocidad de la corriente y la cobertura boscosa. Es necesario seguir adelantando estudios en sistemas acuáticos de la zona del San Juan que permitan entender mejor la dinámica ecológica de los insectos acuáticos y sirvan de base para evaluar el estado ecológico de la cuenca, teniendo en cuenta la presión a la que actualmente está sometida como resultado de la minería y la explotación maderera.

\section{Agradecimientos}

Las autoras agradecen la financiación de la Universidad Tecnológica del Chocó, así como al programa de Jóvenes Investigadores de Colciencias-2007 y al Laboratorio de Limnología de la Universidad Tecnológica del Chocó, por su apoyo en el desarrollo exitoso del presente trabajo.
Agradecemos, igualmente, a la comunidad del corregimiento de Profundo (Tadó-Chocó), por toda su colaboración durante la fase de campo.

\section{Conflicto de intereses}

Las autoras declaran no tener ningún conflicto de intereses.

\section{Bibliografía}

Abril, R.G., Posada, J.A., Parra, S.L.N., Velásquez, C.A., Riss, W., Ospina, T.R. 2004. Los macroinvertebrados acuáticos del páramo de Frontino (Antioquia, Colombia): estudio preliminar. En: Resúmenes XXXI Congreso Sociedad Colombiana de Entomología, SOCOLEN, Bogotá. 75 p.

Adler, P.H., Currie D.C., Wood, D.M. 2004.The black flies (Simuliidae) of North America. Cornell University Press, Ithaca, NY. 941 p.

Alba-Tercedor, J. 1996. Macroinvertebrados acuáticos y calidad de las aguas de los ríos. Departamento de Biología Animal y Ecología, Universidad de Granada. IV Simposio del Agua en Andalucía (SIAGA), Almería, 1996, Vol. II: 203-213.

Allan, J.D. \& Castillo, M.M. 2007. Stream Ecology. Structure and Function of Running Waters. 2a ed. Springer. Dordrecht, Holanda. $436 \mathrm{p}$.

Álvarez, L.F. \& Roldán, G. 1983. Estudio del orden Hemiptera (Heteroptera) en el departamento de Antioquia en diferentes pisos altitudinales. Actualidades Biológicas, 12 (14): 31-46.

Álvarez-Arango, L.F., Arango-Jaramillo, M.C., Roldán-Pérez, G. 2006. Diversidad de los macroinvertebrados dulce acuícolas en Colombia. En: Colombia, Diversa por Naturaleza. Informe Nacional sobre el Avance en el Conocimiento y la Información de la Biodiversidad. Tomo II 19982004. Instituto de Investigación de Recursos Biológicos Alexander Von Humboldt, Bogotá, D. C. 394 p.

APHA-AWWA-WPCF. 1992. Métodos normatizados para el análisis de agua potables y residuales. Traducción del Standard Methods. Díaz de Santos, S. A. (Ed.). Barcelona (España). 385 p.

Arango, M.C., Álvarez, L.F., Arango, G.A., Torres, O.E., Monsalve, A. 2008. Calidad del agua de las quebradas La Cristalina y La Risaralda, San Luis, Antioquia. Revista Escuela de Ingeniería de Antioquia 9: 121-141.

Archangelsky, M., Manzo, V., Michat, M., Torres, P.L.M. 2009. Coleóptera. En: Macroinvertebrados bentónicos sudamericanos. Sistemática y biología. En: Domínguez, E. \& Fernández, H. R. (Eds.). Fundación Miguel Lillo, Tucumán. 411-468 p.

Arias-Díaz, D., Reinoso-Flórez, G., Guevara-Cardona, G.G., Villa-Navarro, F.A. 2007. Distribución espacial y temporal de los coleópteros acuáticos en la cuenca del río Coello (Tolima, Colombia). Caldasia 29 (1): 177-194.

Asprilla, S., Ramírez, J.J., Roldán, G. 1998. Caracterización limnológica preliminar de la ciénaga de Jotaudó (Chocó, Colombia). Actualidades Biológicas 20 (69): 87-107.

Asprilla, S., Mosquera-Murillo, Z., Rivas, M. 2006. Macroinvertebrados acuáticos como indicadores de calidad ecológica del agua en la parte media del río Cabí (Quibdó 
- Chocó). Revista de la Asociación Colombiana de Ciencias Biológicas 18: 43-50.

Bedoya, G., Cediel, F., Restrepo-Correa, I., Cuartas, C., Montenegro, G., Marín-Cerón, M.I. 2009. Aportes al conocimiento de la evolución geológica de las cuencas Atrato y San Juan dentro del arco Panamá-Chocó. Boletín de Geología 31 (2): 61-81.

Bejarano, D., Palacios, E.D., Mosquera-Murillo, Z. 2006. Evaluación de la calidad del agua por medio de la comunidad de trichópteros (Insecta) durante los periodos de aguas altas y bajas en la quebrada La Francisca, Quibdó - Chocó - Colombia. Revista Institucional Universidad Tecnológica del Chocó 25: 59 - 64 .

Cárdenas, A.Y., Bismark, R., López, M., Woo, A., Ramírez, E., Ibrahim, M. 2007. Biodiversidad de macroinvertebrados acuáticos y la calidad del agua en la subcuenca de los ríos Bul Bul y Paiwas, Matiguás, Nicaragua. Encuentro 77: 83-93.

Casas, L., Córdoba, K.E., Asprilla, S., Mosquera-Murillo, Z. 2006 Composición y distribución del orden Ephemeroptera en los ríos Tutunendó y Catugadó, Quibdó-Chocó (Colombia). Libro de la Asociación Colombiana de Limnología 1: 92-97.

Caupaz-Flórez, F., Reinoso, G., Guevara, G., Villa, F.A. 2006. Diversidad y distribución de la familia Elmidae (Insecta: Coleoptera) en la cuenca del río Prado (Tolima, Colombia). Asociación Colombiana de Limnología -Neolimnos 1: 106-116.

Chará, J., Pedraza, G., Giraldo, L., Hincapié, D. 2007. Efecto de los corredores ribereños sobre el estado de quebradas en la zona ganadera del río La Vieja, Colombia. Agroforestería en las Américas 45: 72-78.

Chará-Serna, A.M., Zúñiga, M. del C., Chará, J. 2009. Diversidad de Chironomidae (Insecta: Diptera) asociados a hojarasca en quebradas de la cuenca del río Otún (Risaralda, Colombia). En: Resúmenes del XXXVI Congreso de la Sociedad Colombiana de Entomología. Medellín.

Chará, J., Giraldo, L.P., Zúñiga, M. del C., Chará-Serna, A.M., Pedraza, G.X. 2011. Cambios en el ambienta acuático asociados a la restauración del corredor ribereño en una quebrada afectada por ganadería en la cuenca del río $\mathrm{La}$ Vieja, Colombia. (564-572 p). En: O. Vargas-Ríos \& S.P. Reyes (Eds.). La restauración ecológica en la práctica: Memorias I Congreso Colombiana de Restauración Ecológica y II Simposio Nacional de Experiencias en Restauración Ecológica. Santafé de Bogotá.

Córdoba, K.E., Casas, L., Mosquera-Murillo, Z., Asprilla, S. 2007. Composición y variación temporal del orden Ephemeroptera (Insecta) en los ríos Tutunendó y Catugadó, Quibdó (Chocó - Colombia). Revista de la Asociación Colombiana de Ciencias Biológicas 19: 34-41.

Domínguez, E. 1999. Systematic cladistic and biogeography of the American genus Farrodes (Ephemeroptera, Leptophlebiidae, Atalophlebiinae). Zoological Journal of the Linnean Society of London 126: 155-189.

Domínguez, E., Molineri, C., Pescador, M.I., Hubbard, M.D., Nieto, C. 2006. Ephemeroptera of South América. In: Aquatic Biodiversity en Latin América (ABLA). Adis, J.,
Arias, J. R., Rueda-Delgado, G. \& Wantzen, K. M. (Eds). Vol. 2. Pensoft, Sofia-Moscow.

Domínguez, E. \& Fernández, H.R. 2009 (Eds). Macroinvertebrados bentónicos sudamericanos. Sistemática y biología. Primera Edición. Fundación Miguel Lillo. San Miguel de Tucumán, Argentina. $656 \mathrm{p}$.

Esteves, F. A. 1988. Fundamentos de Limnología. Editorial Interciencias. FINEP. Río de Janeiro, Brasil. 574 p.

Fernández, H. \& Domínguez, E. 2001. Guía para la determinación de los artrópodos bentónicos sudamericanos. Tucumán: Universidad Nacional de Tucumán, Facultad de Ciencias Naturales - Instituto Miguel Lillo. 282 p.

Flowers R. W. \& De La Rosa, C. 2010. Ephemeroptera. En: Macroinvertebrados de agua dulce de Costa Rica I. Springer, M., Ramírez, A. \& Hanson, P. (Eds). Rev. Biol. Trop. 58 (Suppl. 4): 63-93.

Forero-Céspedes, A.M. \& Reinoso-Flórez, G. 2013. Estudio de la familia Baetidae (Ephemeroptera: Insecta) en una cuenca con influencia de la urbanización y agricultura: río Alvarado- Tolima. Revista de la Asociación. Colombiana de Ciencias Biológicas 25: 12-21.

Forero-Céspedes, A. M., Gutiérrez, C., Reinoso-Flórez, G. 2014 nuevos registros de Baetidae (Ephemeroptera: Insecta) para Colombia y el departamento del Tolima. Revista de la Asociación Colombiana de Ciencias Biológicas 26: 59-67.

Giraldo, L.P., Chará, J., Zúñiga, M. del C., Pedraza, G.X., CharáSerna, A.M. 2011. Efectos de los corredores ribereños sobre características bióticas y abióticas de quebradas ganaderas en la cuenca del río La Vieja, Colombia. (pp. 583-591). En: O. Vargas-Ríos \& S.P. Reyes (Eds.). La restauración ecológica en la práctica: Memorias I Congreso Colombiana de Restauración Ecológica y II Simposio Nacional de Experiencias en Restauración Ecológica. Santafé de Bogotá.

Guerrero-Bolaño, F., Manjarrez-Hernández, A., Núñez-Padilla, N. 2003. Los macroinvertebrados bentónicos de Pozo Azul (cuenca del Río Gaira, Colombia) y su relación con la calidad del agua. Acta Biológica Colombiana 8 (2): 43 - 55.

Guevara, G. 2004. Análisis faunístico del orden Trichoptera en su estado larval en la cuenca del río Coello departamento del Tolima. Trabajo de grado (MSc.). Universidad del Tolima, Facultad de Ciencias Básicas, Departamento de Biología, Ibagué.

Guevara-Cardona, G., Reinoso-Flórez, G., Villa-Navarro, F. 2005. Estudio del orden Trichoptera en su estado larval en la cuenca del rio Coello, departamento del Tolima. Revista de la Asociación Colombiana de Ciencias Biológicas 17: 59-70.

Guevara, G., Reinoso, G., Villa, F. 2007. Caddisfly larvae (Insecta: Trichoptera) of the Coello River Basin in Tolima (Colombia): Spatial and temporal patterns and bioecological aspects. Págs. 8-12 en: J. Bueno-Soria, R. Barba-Álvarez and B. Armitage (Eds), Proceedings of the XIIth International Symposium on Trichoptera. The Caddis Press. Columbus, Ohio.

Hammer, O. Dat Harper, D.A.T., Ryan, P.D. 2001. Past: Paleontological Statistics Software Package for Education and Data Analysis. Palaeontologia Electronica 4. Disponible en: http://palaeoelectronica.org/2001_1/past/issue1_01.htm. 
Hammond, J.I., Luttbeg, B., Sih, A. 2007. Predator and prey space use: Dragonflies and tadpoles in an interactive game. Ecology 88: 1525-1535.

Hanson, P., Springer, M., Ramírez, A. 2010. Introducción a los grupos de macroinvertebrados acuáticos. En: Macroinvertebrados de agua dulce de Costa Rica I. Springer, M., Ramírez, A. \& Hanson, P. (Eds). Rev. Biol. Trop. 58 (Suppl. 4): 3-37.

Hawkins, C.P., Murphy, M.M., Anderson, N.H. 1982. Effects of canopy, substrate composition, and gradient on the structure of macroinvertebrate communities in Cascade Range streams of Oregon. Ecology 63: 1840-1856.

Holdridge, R.L. 1996. Ecología basada en zonas de vida. Instituto Interamericano de Cooperación para la Agricultura (IICA). San José de Costa Rica.

Instituto de Investigaciones Ambientales del Pacífico - IIAP. 2013. Proyecto Plan estratégico de la macro cuenca del Pacífico Colombiano. Quibdó-Chocó. Informe Final. 487 p.

Latorre, I., Montaño, M., Rincón, M. 2006. Comunidad de insectos acuáticos del río Dulce (Villeta, Cundinamarca). Rev UDCA. Actual. Divulg. Cient 9 (1): 151-161.

Lombardo, P. 1997. Predation by Enallagma nymphs (Odonata, Zygoptera) under different conditions of spatial heterogeneity. Hydrobiologia 356: 1-9.

Machado, T. \& Roldán, G. 1981. Estudio de las características fisicoquímicas y biológicas del río Anori y sus principales afluentes. Rev. Actual. Biol. 10 (35): 3-19.

Malmqvist, B., Adler, P.H., Kuusela, K., Merritt, R.W., Wooton, R.S. 2004. Black flies in the boreal biome, key organisms in both terrestrial and aquatic environments: A review. Ecoscience 11: 187-200.

Margalef, R. 1998. Ecología. Novena edición. Ediciones Omega. Barcelona, España. 951 p.

Mazzucconi, S.A., López R.M., Bachmann, A.O. 2009. Hemiptera- Heteroptera: Gerromorpha y Nepomorpha. En: Macroinvertebrados bentónicos sudamericanos: Sistemática y Biología. Domínguez, E. \& Fernández, H. R. (Eds). Primera edición. Fundación Miguel Lillo. San Miguel de Tucumán, Argentina. 168-231 p.

Merritt, R.W. \& Cummins, K.W. 1996. An introduction to the aquatic insects of North America. Third edition. Dubuque: Kendall/Hunt Publishing Company, Dubuque, Iowa. 862 p.

Molano, F. \& Camacho, D.L. 2005. Especies de Gerridae (Heteroptera: Gerromorpha) del departamento del Quindío. En: Riqueza Biótica Quindiana. Armenia, Quindío. Agudelo, C. (Ed). Ed. OPTIGRAF. (Colombia). 370-376 p.

Mosquera, S., Zúñiga, M. del C., Guevara, G. 2002. Diversidad y distribución de coleópteros acuáticos y semiacuáticos con énfasis en la familia Elmidae en el suroccidente colombiano. En: Resúmenes V Seminario Colombiano de Limnología y I Reunión Internacional de Limnología del alto Amazonas. Leticia, Colombia.

Mosquera, Z. \& Bejarano, D. 2006. Estudio del orden Trichoptera (Insecta) en dos ecosistemas lóticos del municipio de Quibdó, Chocó-Colombia. Pág. 14 en: F. Villa, C. Rivera, G.
Reinoso \& M. Núñez (Eds), Resúmenes del VII Seminario Colombiano de Limnología y I Reunión Internacional sobre Ríos y Humedales Neotropicales. Asociación Colombiana de Limnología, Ibagué.

Mosquera, Z., Bejarano, D., Asprilla, S. 2006. Estudio del orden Trichoptera (Insecta) en dos ecosistemas lóticos del municipio de Quibdó, Chocó - Colombia. Libro de la Asociación Colombiana de Limnología 1: 85-91.

Muñoz, F. 2004. El orden Trichoptera (Insecta) en Colombia: II inmaduros y adultos, consideraciones generales. En: Fernández, F., Andrade, M. G. \& Amat, G. (Eds.). Insectos de Colombia. Universidad Nacional de Colombia, Facultad de Ciencias. Santafé de Bogotá, Colombia 3: 319-349.

Ocón, C.S. \& Rodríguez, A. 2004. Presence and abundance of Ephemeroptera and other sensitive macroinvertebrates in relation with habitat conditions in pampean streams (Buenos Aires, Argentina). Arch. Hydrobiol 159: 473-487.

Ometo, J.P., Matinelli, L.A, Ballester, M.B., Gessner, A., Krusche, A.V., Victoria, R.L., Williams, M. 2000. Effects of land use water chemistry and macroinvertebrate in two streams of the Piracicaba river basin, south-east Brazil. Freshwater Biol 44: 327-337.

Padilla-Gil, D.N. \& Arcos, O. 2011. Hemiptera acuáticos asociados a los estuarios de la Costa Pacífica Colombiana. Revista Colombiana de Entomología 37 (1): 350-353.

Parra-Trujillo Y.T., Padilla Gil, D.N., Reinoso-Flórez, G. 2014. Diversidad y distribución de Rhagovelia (Hemiptera, Veliidae) del departamento del Tolima. Revista de la Asociación Colombiana de Ciencias Biológicas 26: 82-88.

Posada, G.J. \& Roldán, G. 2003. Clave ilustrada y diversidad de las larvas de Trichoptera en el noroccidente de Colombia. Caldasia 25 (1): 169-192.

Ramírez, A. 2010. Odonata. En: Macroinvertebrados de agua dulce de Costa Rica. Springer, M., Ramírez, A. \& Hanson, P. (Eds.) Revista Biología Tropical 58 (Supl 4): 97-136.

Reinoso, G., Villa, F., Esquivel, H., García, J., Vejarano, M. 2007. Biodiversidad faunística y florística de la cuenca del río Totaré - Biodiversidad regional fase III. Informe final. Universidad del Tolima. Ibagué.

Reinoso, G., Guevara, G., Vejarano, M., García, J., Villa, F. 2008. Áreas de interés para la conservación en la cuenca del río Prado (Tolima, Colombia): macroinvertebrados y calidad de agua como factores clave. Revista de la Asociación Colombiana de Ciencias Biológicas 20: 102-116.

Ribera, I., Hogan, J.E., Vogler, A.P. 2002. Phylogeny of hydradephagan water beetles inferred from 18S rRNA sequences. Molecular Phylogenetics and Evolution 23: 43-62.

Richoux, P. 1994. Theoretical hábitat templets, species traits, and species richness: Aquatic Coleoptera in the Upper Rhone River and its floodplain. Freshwater Biology 31: 377-395.

Rivas, M., Mosquera, Z., Asprilla, S. 2003. Calidad ecológica de las aguas de la parte media del río Cabí (Quibdó-Chocó) utilizando los macroinvertebrados como indicadores. Revista Institucional Universidad Tecnológica del Chocó 19: 59-63. 
Rivera-Usme, J.J., Camacho-Pinzón, D.L., Botero-Botero, A. 2008. Estructura numérica de la entomofauna acuática en ocho quebradas del departamento del Quindío-Colombia. Acta biol. Colomb. 13 (2): 133 - 146.

Rojas, M.R., Molano, F., Morales, I.T. 2006. Contribución al conocimiento de los chinches semiacuáticos (Hemiptera: Gerridae) en ambientes lóticos y lénticos del departamento de Risaralda. Revista Investigaciones Universidad del Quindío 16: 37-47.

Roldán, G.A. 1992. Fundamentos de limnología neotropical. Medellín (Antioquia): Universidad de Antioquia. 592 p.

Roldán, G.A. 1996. Guía para el estudio de los macroinvertebrados acuáticos del Departamento de Antioquía. FEN-Colombia, COLCIENCIAS-Universidad de Antioquia, Medellín. 234 p.

Roldán, G.A. 2003. Bioindicación de la calidad del agua en Colombia: uso del método BMWP/Col. Editorial Universidad de Antioquia. Primera edición. 170 p.

Romero, B.I., Pérez, S.M., Rincón, M.H. 2006. Ephemeroptera del Parque Nacional Natural "Cueva de los Guácharos", Huila, Colombia. Rev UDCA Actual. Divulg. Cient. 9 (1): 141-149.

Rosenberg, D.M. \& Resh, V.H. 1993a. Introduction to freshwater biomonitoring and benthic macroinvertebrates, p. 1-9. En: D.M. Rosenberg, V.H. Resh (Eds.). Freshwater biomonitoring and benthic macroinvertebrates. Chapman \& Hall, Nueva York, EEUU.

Rosenberg, D.M. \& Resh, V. 1993b. Rapid assessment approaches to biomonitoring using benthic macroinvertebrates, $p$. 195-233. En: V. Resh, H.K. Jackson (Eds.). Freshwater biomonitoring and benthic macroinvertebrates. Chapman \& Hall, Londres, Inglaterra.

Salas, Y., Geovo, S., Asprilla, S. 2011. Caracterización de las comunidades perifíticas y de macroinvertebrados acuáticos presentes en el río Pacurita, corregimiento de Pacurita, Quibdó-Chocó-Colombia. Revista Biodiversidad Neotropical 2: 98-104.

Shieh, S.H. \& Yang, P.S. 2000. Community structure and functional organization of aquatic insects in an agricultural mountain stream of Taiwan: 1985-1986 and 1995-1996. Zool. Stud. 39: 191-202.

Springer, M., Ramírez, A., Hanson, P. 2010. Macroinvertebrados de agua dulce de Costa Rica I. Rev. Biol. Trop. 58 (Suppl. 4): 97-136.

Tamaris-Turizo, C., Turizo-Correa, R., Zúñiga, M.C. 2007. Distribución espacio-temporal y hábitos alimentarios de ninfas de Anacroneuria (Insecta: Plecoptera: Perlidae) en el río Gaira (Sierra Nevada de Santa Marta, Colombia). Caldasia 29 (2): 375-385.
Torres, Y., Roldan, G., Asprilla, S., Rivas, T. 2006. Estudio preliminar de algunos aspectos ambientales y ecológicos de las comunidades de peces y macroinvertebrados acuáticos en el río Tutunendó, Chocó-Colombia. Rev. Acad. Colomb. Cienc. 30 (114): 67-76.

Vásquez-Ramos, J.M., Ramírez-Díaz, F., Reinoso-Flórez, G. 2010. Distribución espacial y temporal de los tricópteros inmaduros en la cuenca del río Totaré (Tolima-Colombia). Caldasia 32 (1): 129-148.

Vásquez-Ramos, J.M. \& Reinoso, G. 2012. Estructura de la fauna béntica en corrientes de los Andes colombianos. Revista Colombiana de Entomología 38 (2): 351-358.

Vásquez-Ramos, J.M., Guevara-Cardona, G., Reinoso-Flórez, G. 2014. Factores ambientales asociados con la preferencia de hábitat de larvas de tricópteros en cuencas con bosque seco tropical (Tolima, Colombia). Revista de Biología Tropical 62 (2): 21-40.

Wallace, I.B. \& Webster, J.R. 1996.The role of macroinvertebrates in stream ecosystem function. Ann. Rev. Ecol. Syst. 17: $567-594$.

Walteros-Rodríguez, J.M. \& Paiba-Alzate, J.E. 2010. Estudio preliminar de la comunidad de macroinvertebrados acuáticos en la reserva forestal torre cuatro. Bol. Cient. Mus. Hist. Nat 14 (1): 137 - 149.

Wotton, R.S. \& Malmqvist, B. 2001. Feces in aquatic ecosystems. Bioscience 51: 537-544.

Zamora, G.H. 2002. Análisis biogeográfico de los macroinvertebrados acuáticos epicontinentales en el departamento del Cauca, Colombia. Rev Asoc Col Cienc. 14 (1): 37-51.

Zúñiga, M.C., Molineri, C., Domínguez, E. 2004. Orden Ephemeroptera (Insecta) de Colombia En: Insectos de Colombia. Fernández, F., Andrade, M. G. \& Amat, G. (Eds.). Universidad Nacional de Colombia, Facultad de Ciencias. Santafé de Bogotá, Colombia. 3: 17- 42.

Zúñiga, M.C. 2010. Diversidad, distribución y ecología del orden Plecoptera (Insecta) en Colombia, con énfasis en Anacroneuria (Perlidae). Momentos de Ciencia 7: 101-112.

Zúñiga, M. del C., Chará, J.C., Giraldo, L.P., Chará-Serna, A.M., Pedraza, G.X. 2013. Composición de la comunidad de macroinvertebrados acuáticos en pequeñas quebradas de la región andina colombiana, con énfasis en la entomofauna. Dugesiana 20 (2): 263-277.

Zúñiga M.C., Cardona, W., Molineri, C., Mendivil, J., Cultid, C., Chará, A.M. \& Giraldo, A. 2014. Entomofauna Acuática del Parque Nacional Natural Gorgona, Pacífico colombiano, con énfasis en Ephemeroptera y Plecoptera. Rev. Biol. Trop. 62 (Suppl. 1): 221-241. 